\title{
ON THE DIFFERENCE OF CONSECUTIVE PRIMES
}

\author{
P. ERDÖS
}

The present paper contains some elementary results on the difference of consecutive primes. Theorem 2 has been announced in a previous paper. ${ }^{1}$ Also some unsolved problems are stated.

Let $p_{1}=2, p_{2}=3, \cdots, p_{k}, \cdots$ be the sequence of consecutive primes. Put $d_{k}=p_{k+1}-p_{k}$. We have:

TheOReM 1. There exist positive real numbers $c_{1}$ and $c_{2}, c_{1}<1, c_{2}<1$, such that for every $n$ the number of $k$ 's satisfying both

$$
d_{k+1}>\left(1+c_{1}\right) d_{k},
$$$$
k \leqq n,
$$

and the number of l's satisfying both

$$
d_{l+1}<\left(1-c_{1}\right) d_{l}, \quad l \leqq n,
$$

are each greater than $c_{2} n$.

We shall prove Theorem 1 later. From Theorem 1 we easily deduce:

THEOREM 2. For every $t$ and all sufficiently large $n$ the number of solutions in $k$ and $l$ of each of the two sets of inequalities

$$
\left(\frac{p_{k+1}^{t}+p_{k-1}^{t}}{2}\right)^{1 / t}>p_{k}, k \leqq n ; \quad\left(\frac{p_{l+1}^{l}+p_{l-1}^{t}}{2}\right)^{1 / t}<p_{l}, l \leqq n,
$$

is greater than $\left(c_{2} / 2\right) n$.

Let $\epsilon$ be sufficiently small but fixed. It is well known that $p_{n}<2 \cdot n$ $\log n$. Thus the number of $k \leqq n$, with $p_{k+1}>(1+\epsilon) p_{k}$, is less than $c \log n$. Hence it follows from Theorem 1 that the number of $k$ 's satisfying

$$
p_{k+1}<(1+\epsilon) p_{k}, \quad d_{k}>\left(1+c_{1}\right) d_{k-1}, \quad k \leqq n,
$$

is greater than $\left(c_{2} / 2\right) n$. A simple calculation now shows that the primes satisfying (4) also satisfy the first inequality of (3) if $\epsilon=\epsilon\left(c_{1}\right)$ is chosen small enough. The second inequality of (3) is proved in the same way, which proves Theorem 2 .

Further, we obtain, as an immediate corollary of Theorem 1, that ${ }^{2}$

Received by the editors October 17, 1947.

${ }^{1} \mathrm{P}$. Erdös and P. Turán, Some new questions on the distribution of primes, Bull. Amer. Math. Soc. vol. 54 (1948) pp. 371-378.

2 This result was also stated in the above paper. 


$$
\lim \sup d_{k+1} / d_{k}>1, \quad \lim \inf d_{k+1} / d_{k}<1 .
$$

At present I can not decide whether $d_{k+2}>d_{k+1}>d_{k}$ has infinitely many solutions. The following question might be of some interest: Let $\epsilon_{n}=1$ if $d_{n+1}>d_{n}$, otherwise $\epsilon_{n}=0$. It may be conjectured that $\sum_{n=1}^{\infty} \epsilon_{n} / 2^{n}$ is irrational. I can not even prove that from a certain point on $\epsilon_{n}$ is not alternatively 1 and 0 .

In order to prove Theorem 1 we need two lemmas.

LEMMA 1. For sufficiently small $c_{1}>0$ the number of solutions in $k$ of the inequalities

$$
1+c_{1}>d_{k+1} / d_{k}>1-c_{1}, \quad k \leqq n,
$$

is less than $n / 4$.

Denote by $g(n ; a, b)$ the number of solutions of the simultaneous equations

$$
d_{k+1}=a, \quad d_{k}=b, \quad k \leqq n .
$$

Denote by $V$ the number of primes $r<2 \cdot n \cdot \log n$ for which $r+a$ and $r+a+b$ are also primes. Since $p_{n}<2 \cdot n \cdot \log n$, we evidently have

$$
g(n ; a, b) \leqq V .
$$

Now let $c_{1}>0$ be sufficiently small and $q_{1}, q_{2}, \cdots$ run through the primes less than $n^{c_{3}}$. Then $V$ is not greater than $n^{c_{3}}$ plus the number $U$ of integers $m<2 \cdot n \cdot \log n$, which satisfy, for all $i$,

$$
m \neq \equiv 0\left(\bmod q_{i}\right), \quad m \neq \equiv-a\left(\bmod q_{i}\right), \quad m \not \equiv-(a+b)\left(\bmod q_{i}\right) .
$$

If $q \nmid a \cdot b \cdot(a+b)$ then these three residues are all different. In a previous paper ${ }^{3}$ I stated the following theorem: Let $q_{1}, q_{2}, \cdots$ be primes all less than $n^{c_{3}}$. Associate with each $q_{i} t$ distinct residues $r_{1}^{(i)}, \cdots, r_{i}^{(i)}$. Then the number of integers $m \leqq n$ for which

$$
m \neq r_{j}^{(i)}\left(\bmod q_{i}\right), \quad j=1,2, \cdots, t ; i=1,2, \cdots,
$$

is less than

$$
\operatorname{cn} \prod_{i}\left(1-\frac{t}{q_{i}}\right)
$$

The proof of this theorem follows easily from Brun's method. ${ }^{3}$ Thus

${ }^{8}$ P. Erdös, Proc. Cambridge Philos. Soc. vol. 33 (1937) p. 8, Lemma 2. A book of Rosser and Harrington on Brun's method will soon appear which will contain a detailed proof of this result. 
we have

$$
U<c_{4} n \log n \prod_{q}\left(1-\frac{3}{q}\right), \quad q<n^{c_{3},} \quad q\{a \cdot b \cdot(a+b) .
$$

It is well known that

$$
\prod_{q<x}\left(1-\frac{3}{q}\right)<\frac{c}{(\log x)^{3}} \text { and } \prod_{q}\left(1-\frac{q}{q^{2}}\right)>0 \text {. }
$$

Thus

$$
U<c_{5} \frac{n}{(\log n)^{2}} \prod_{q}\left(1+\frac{3}{q}\right), \quad q \mid a \cdot b \cdot(a+b) .
$$

Hence finally from (6) and $V \leqq U+n^{c_{3}}$,

$$
g(n ; a, b)<c_{5}^{\prime} \frac{n}{(\log n)^{2}} \prod_{q}\left(1+\frac{3}{q}\right), \quad q \mid a \cdot b \cdot(a+b) .
$$

Now we split the $k$ 's satisfying (5) into two classes. In the first class put the $k$ 's with $d_{k}>20 \log n$ and in the second class the other $k$ 's. From $p_{n}<2 \cdot n \cdot \log n$ we deduce that the number of $k$ 's of the first class is less than $n / 10$.

The number of the $k$ 's of the second class is not greater than

$$
\text { (8) } \quad \sum^{\prime} g(n ; a, b)<c_{5}^{\prime} \frac{n}{(\log n)^{2}} \sum^{\prime} \prod_{q}\left(1+\frac{3}{q}\right), \quad q \mid a \cdot b \cdot(a+b) \text {, }
$$

where the prime indicates that the summation is extended over those $a$ and $b$ with $a<20 \cdot \log n, 1+c_{1}>b / a>1-c_{1}$. Now

$$
\sum^{\prime} \prod_{q \mid a \cdot b(a+b)}\left(1+\frac{3}{q}\right) \leqq \sum_{1}\left(\prod_{q \mid a}\left(1+\frac{3}{q}\right) \sum_{2} \prod_{q \mid b(a+b)}\left(1+\frac{3}{q}\right)\right.
$$

where in $\sum_{1}, a<20 \log n$ and in $\sum_{2}, 1+c_{1}>b / a>1-c_{1}$. We have

$$
\begin{aligned}
\sum_{2} \prod_{q \mid b(a+b)}\left(1+\frac{3}{q}\right) & <\sum_{2}\left(\prod_{q \mid b}\left(1+\frac{3}{q}\right)^{2}+\prod_{q \mid a+b}\left(1+\frac{3}{q}\right)^{2}\right) \\
& <\sum_{2}\left(\prod_{q \mid b}\left(1+\frac{15}{q}\right)+\prod_{q \mid a+b}\left(1+\frac{15}{q}\right)\right) \\
& <\sum_{m<3 a} 2\left(1+\frac{2 c_{1} a}{m}\right) \frac{15^{V(m)}}{m}<c_{6} c_{1} a,
\end{aligned}
$$

4 See, for example, Hardy-Wright, p. 349. 
by interchanging the order of summation and by observing that the number of $b$ 's satisfying $1+c_{1}>b / a>1-c_{1}$ and $b \equiv 0(\bmod m)$ is less than $1+\left(2 \cdot c_{1} \cdot a / m\right)$. The same holds for the $b$ 's satisfying $1+c_{1}$ $>b / a>1-c_{1}$ and $a+b \equiv 0(\bmod m) .(v(m)$ denotes the number of prime factors of $m$.) Thus

$$
\begin{aligned}
\Sigma^{\prime \prime} \prod_{q \mid a \cdot b \cdot(a+b)}\left(1+\frac{3}{q}\right) & <c_{6} c_{1} \sum_{1} a \prod_{q \mid a}\left(1+\frac{3}{q}\right) \\
& <20 c_{6} c_{1} \log n \sum_{1} \prod_{q \mid a}\left(1+\frac{3}{q}\right) \\
& <20 c_{6} c_{1} \log n \sum_{m=1}^{\infty} \frac{(20 \log n) 3^{V(m)}}{m^{2}} \\
& <c_{7} c_{1}(\log n)^{2}<\frac{1}{10 c_{5}^{\prime}}(\log n)^{2}
\end{aligned}
$$

if $c_{1}<1 / 10 \cdot c_{7} \cdot c_{5}^{\prime}$. Hence finally from (8) the number of solutions of (5) is less than

$$
n / 10+n / 10<n / 4
$$

which proves Lemma 1.

LEMMA 2. There exists a constant $c_{8}$ so that the number of integers $k \leqq n$ satisfying

$$
d_{k+1} / d_{k}>t \text { or } \quad d_{k+1} / d_{k}<1 / t
$$

is less than $c_{8} \cdot n / t^{1 / 2}$.

It suffices to prove the lemma for large $t$. We split the integers $k$ satisfying (9) into two not necessarily disjoint classes. In the first class are the $k$ 's for which either

$$
d_{k} \geqq t^{1 / 2} \cdot \log n \quad \text { or } \quad d_{k+1} \geqq t^{1 / 2} \cdot \log n .
$$

In the second class are the $k$ 's for which either

$$
d_{k} \leqq(\log n) / t^{1 / 2} \text { or } \quad d_{k+1} \leqq(\log n) / t^{1 / 2} .
$$

Clearly if (9) is satisfied then $k$ is in one of these classes.

We obtain from $p_{n}<2 \cdot n \cdot \log n$ that the number of $k$ 's of the first class is less than $4 \cdot n / t^{1 / 2}$.

As in the proof of Lemma 1 we obtain from our result proved in a previous paper ${ }^{3}$ that the number $Z$ of solutions of $d_{u}=a, u \leqq n$ is less than 


$$
Z<c_{9} n \log n \prod_{q}\left(1-\frac{2}{q}\right), \quad q \nmid a, q<n^{c_{3} .}
$$

Thus as in Lemma 1

$$
Z<c_{10} \frac{n}{\log n} \prod_{p \mid a}\left(1+\frac{2}{p}\right) .
$$

Thus the number of $k$ 's of the second class is less than

$$
\begin{aligned}
2 c_{10} \frac{n}{\log n} \sum_{a<\log n / t^{1 / 2}} \prod_{p \mid a}\left(1+\frac{2}{p}\right) & <2 c_{10} \frac{n}{\log n} \sum_{p=1}^{\infty} \frac{(\log n) 2^{V(d)}}{t^{1 / 2} d^{2}} \\
& <\frac{c_{11} n}{t^{1 / 2}},
\end{aligned}
$$

which proves Lemma 2, with $c_{8}=2+c_{11}$.

Now we can prove Theorem 1. It will suffice to prove (1). Suppose that (1) is not true. Then for every $c_{1}>0$ and $\epsilon>0$ there exists an arbitrarily large $n$ so that the number of solutions of

$$
d_{k+1}>\left(1+c_{1}\right) d_{k}
$$

is less than $\epsilon \cdot n$. Consider the product

$$
\frac{d_{n}}{d_{1}}=\frac{d_{2}}{d_{1}} \cdot \frac{d_{3}}{d_{2}} \cdots \frac{d_{n}}{d_{n-1}} .
$$

By Lemma 2 the number of $k \leqq n$ satisfying $d_{k+1} / d_{k}>2^{2 l}$ is less than $c_{8} n / 2^{l}$. Thus by Lemma 1 and (10) we have for every $u$

$$
\begin{aligned}
d_{n} / d_{1} & <2^{2 u \epsilon n} \prod_{l \geqq 2^{2 u}}\left(2^{2 l}\right)^{c_{8} n / 2 l} \cdot\left(1+c_{1}\right)^{n / 4}\left(1-c_{1}\right)^{n / 2} \\
& <2^{2 u \epsilon n} \exp \sum_{l \geqq u} \frac{c_{8} l n \log 4}{2^{l}} \cdot\left(1-c_{1}\right)^{n / 4}
\end{aligned}
$$

If $\epsilon$ is sufficiently small there is a suitable choice of $u$ such that $2^{2 u \in n}<\left(1+c_{1}\right)^{n / 8}$ and

$$
\exp \sum_{l \geqq u} \frac{c_{8} \ln \log 4}{2^{l}}<\left(1+c_{1}\right)^{n / 8} .
$$

Thus $d_{n} / d_{1}<\left(1-c_{1}^{2}\right)^{n / 4}<1 / n$ for arbitrarily large $n$, an evident contradition. This proves (1) and completes the proof of Theorem 1.

SyRACUSE UNIVERSITY 